\title{
Ueber einen histonähnlichen Körper aus Thymus. \\ Von
}

A. Fleroff.

(Aus dem physiologischen Institut in Marburg.)

(Der Redaction zugegangen am 21. Juli 1899.)

Nachdem durch die Untersuchungen von A. Kossel1) eine engere Beziehung des Histons zu den Protaminen wahrscheinlich gemacht worden ist, erhebt sich die Frage, ob in gewissen thierischen Geweben, die eine reichliche Menge von Histon enthalten, neben diesem auch Protamin nachgewiesen werden kann.

Auf Veranlassung von Prof. A. Kossel habe ich zur Untersuchung dieser Frage Thymusgewebe mit denjenigen Methoden behandelt, welche ron demselben ${ }^{2}$ ) für die Gewinnung des Protamins aus Fischsperma angegeben sind.

Zunächst verfuhr ich in folgender Weise. Die zerhackte Thymusdrüse wurde mit Alkohol und Aether erschöpft und sodann 48 Stunden lang mit einer $2 \%$ igen Schwefelsäure (auf je $100 \mathrm{gr}$. Thymusdrüse $1000 \mathrm{ccm}$. Schwefelsäure) extrahirt und der so gewonnene Auszug mit der dreifachen Menge 96\% igen Alkohols versetzt. Fs entstand ein Niederschlag, welcher im heissen Wasser gelöst und mit pikrinsaurem Natrium in das Pikrat übergeführt wurde.

1) A. Kossel, Ueber einen peptonartigen Bestandtheil des Zellkerns. Diese Zeitschrift, Bd. VIII, 1884, S. ó11. Deutsche medicinische Wochenschrift, Jahrgang 1894, Nr. 7, 1898, Nr. 37.

2) A. Kossel, Ueber die Constitution der einfachsten Eiweissstoffe (Diese Zeitschrift, Bd. XXV, 1898). 
Das Pikrat wurde sodann mittels 2\% $\%$ iger Schwefelsäure und Aether von der Pikrinsäure befreit und die Lösung von Neuem mit Alkohol gefällt. Diese Fällung wurde behufs weiterer Reinigung zweimal wiederholt. Die auf diese Art erhaltene Substanz stellte ein weisses Pulver dar. Um aus diesem Sulfat die freie Base zu erhalten, wurde der Niederschlag in heissem Wasser gelöst und mit Baryumhydrat im Ueberschuss versetzt. Das überschüssige Baryumhydrat wurde mit Kohlensäure entfernt. Es entstand eine trübe, schlecht filtrirende Lösung. Die Filtration wurde erst möglich, als ich das gleiche Volumen Alkohol und etwas Ammoniak zufügte und nun erwärmte. Das klare Filtrat wurde mit einem Ueberschuss von Alkohol versetzt, wodurch ein schwachgelber Niederschlag erhalten wurde. Derselbe wurde nun durch zweimaliges Auflösen in heissem Wasser und Versetzen mit Alkohol unter Zusatz von Aether gereinigt. Der so erhaltene Körper hatte folgende Eigenschaften.

Die wässerige Lösung desselben reagirte bei gänzlicher Abwesenheit von Ammoniak deutlich alkalisch. Ammoniak fällte ihn in wässeriger Lösung selbst im Ueberschuss nicht. Alkohol, im Ueberschuss zugesetzt, bewirkte eine Fällung, die aber schwierig erfolgte und anscheinend unvollständig war; Zusatz von Aether erleichterte diese Fällung. Alkohol, dem eine kleine Menge Ammoniak zugesetzt war, fällte den Körper vollständig. Der Körper gibt die Biuretreaction. Millon's Reagens erzeugte eine kaum wahrnehmbare Rosafärbung. Phosphorwolframsäure, Gerbsäure, Ferrocyankalium mit Essigsäure, pikrinsaures Natrium, Jodquecksilber-Jodkalium, letztere beiden in neutraler oder schwach alkalischer Lösung, fällen den Körper. Jodjodkalium, chromsaures Natrium, Sublimat, Steinsalz (sogar gesättigte Lösung), Zinksulfat (gesättigte Lösung) bewirkten keine Fällung. Albumose in alkalischer Lösung fällte auch nicht. Der Körper gab keine Farbenreaction mit Eisessig und concentrirter Schwefelsäure.

Durch Alkali und kohlensaures Natrium entstand ein Niederschlag, der sich im Ueberschuss des Fällungsmittels schnell löste. Salpetersäure bewirkte keine Fällung. Beim 
Kochen der wässerigen Lösung war keine Coagulation bemerkbar, auch nicht auf Zusatz von kohlensaurem Natron. Der erhaltene Körper wurde bei $100^{\circ} \mathrm{C}$. bis zur Gewichtsconstanz getrocknet und analysirt. Die Analyse ergab:

$0,2231 \mathrm{~g}$ der Substanz gaben $0,4235 \mathrm{CO}_{2}$ und $0,1610 \mathrm{H}_{2} \mathrm{O}$

$0,1583 \mathrm{~g} \gg \quad$ 》 $24,6 \mathrm{ccm}$. $\mathrm{N}$ bei $18^{\circ} \mathrm{C}$. und $750,5 \mathrm{~mm}$ Bar.

$0,3752 \mathrm{~g}$ 》 " $0,05022 \mathrm{~g} \mathrm{BaSO}_{4}, 0,00689 \mathrm{~g} \mathrm{~S}$.

Beim Verbrennen auf Platinblech war keine Asche bemerkbar.

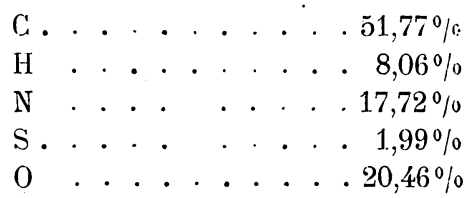

Da bei der eben beschriebenen Darstellungsweise die Ausbeute sehr gering war, so habe ich es versucht, ein zweites Verfahren zur Gewinnung dieser Substanz ausfindig zu machen. $\mathrm{Zu}$ dem Zwecke habe ich die heisse Lösung des Sulfats nicht mit Barythydrat zersetzt, sondern nach vorausgegangener Ueberführung in pikrinsaures Natrium und Rückverwandlung in das Sulfat dieses mit Ammoniak im Ueberschuss gefällt. Hierbei war ich in Folge freundlicher Mittheilungen des Herrn Dr. Levene in der Lage, das folgende, noch nicht publicirte Verfahren zu benutzen, welches Herr Dr. Levene im hiesigen Laboratorium zur Trennung von Histon und Protamin ausgearbeitet hat. Die warme Lösung wurde von dem durch Ammoniak erhaltenen Niederschlag abfiltrirt, der Niederschlag dreimal mit heissem Wasser gewaschen und die ablaufenden Wässer mit dem ersten Filtrat vereinigt. Das Filtrat wurde nun mit einem Ueberschuss von Alkohol gefällt und der Niederschlag in heissem Wasser gelöst. Beim Hinzufügen einer geringen Menge Alkohol zu dieser Lösung entstand eine Trübung; dieselbe wurde abfiltrirt und das Filtrat mit einem Ueberschuss von Alkohol unter Zusatz von Aether gefällt. ${ }^{1}$ ) Der so erhaltene, durch wässeriges Ammoniak und durch wenig Alkohol nicht fällbare, durch

1) Diese Fällung mit Ammoniak und die nachfolgende Behandlung mit Alkohol, sowie die Fällung mit Aetheralkohol wurde viermal wiederholt. 
Alkoholäther fällbare Körper entsprach in allen Reactionen dem Körper, der nach der ersten Methode erhalten wurde, doch war die Ausbeute fast zweimal so gross. Der Körper wurde bei $125^{\circ} \mathrm{C}$. bis zur Gewichtsconstanz getrocknet und analysirt. Die Analyse hatte folgendes Ergebniss:

$0,2393 \mathrm{~g}$ der Substanz gaben 0,455 ó $\mathrm{CO}_{2}$ und $0,1664 \mathrm{H}_{2} \mathrm{O}$

$0,2736 \mathrm{~g} » \quad » 43 \mathrm{ccm}$. $\mathrm{N}$ bei $17,5^{\circ} \mathrm{C}$. und $749 \mathrm{~mm}$ Bar.

$$
\begin{aligned}
& \text { C. . . . . . . } 51,91 \% \\
& \mathrm{H} \text {. . . . . . . . 7,81\% } \\
& \mathrm{N} \text {. . . . . . . 17,97\% } \\
& \mathrm{O} \text {. . . }(20,32 \%) \text {. Nach Abzug von 1,99\%) S }
\end{aligned}
$$

Der Schwefelgehalt wurde nicht bestimmt.

Demnach stimmt der so erhaltene Körper mit dem ersten in der Zusammensetzung überein.

I

$$
\begin{array}{llll}
\mathrm{C} & \ldots & . & 51,77 \% \\
\mathrm{H} & \cdots & . & 8,06 \% \\
\mathrm{~N} & . & . & 17,72 \% \\
\mathrm{O} & . & . & (20,46 \%)
\end{array}
$$

II. $\check{51,91 \%}$ $7,81 \%$ $17,97 \%$ $(20,32 \%)$.

Der durch Ammoniak entstandene Niederschlag wurde mit heissem Wasser sorgfältig ausgewaschen und in einem kleinen Ueberschuss von verdünnter Salzsäure gelöst. Dieser Körper gab alle für das Histon ${ }^{1}$ ) typischen Reactionen, er war in Ammoniak vollständig unlöslich, wurde durch Salpetersäure in der Kälte gefällt, aber nicht beim Erwärmen, durch kohlensaures Natrium wurde er coagulirt, zeigte deutlich die Millon'sche Reaction, löste sich gar nicht beim Fällen mit Ammoniak im heissen Wasser und gab auch andere weniger charakteristische Histonreactionen. Aus der salzsauren Lösung wurde der Körper mit einem Ueberschuss von Ammoniak gefällt und der Niederschlag so lange mit heissem Wasser ausgewaschen, bis das ablaufende Wasser keinen Gehalt an Chlor und Ammoniak mehr zeigte. Der bei $100^{\circ} \mathrm{C}$ bis zur Gewichts-

1) A. Kossel (loco citato).

Lilienfeld, Zur Chemie der Leucocyten (Diese Zeitschrift, Bd. XVIII, 1894, S. 473.) 
constanz getrocknete Körper wurde analysirt. Er stellt ein weisses Pulver dar. Die Analyse gab folgende Werthe:

$0,2234 \mathrm{~g}$ der Substanz gaben $0,4290 \mathrm{CO}_{2}$ und $0,1550 \mathrm{H}_{22} \mathrm{O}$

$0,2248 \mathrm{~g} " \quad \Rightarrow 36,8 \mathrm{ccm} . \mathrm{N}$ bei $17^{\circ} \mathrm{C}$. und $750 \mathrm{~mm} . \mathrm{B}$.

$0,4705 \mathrm{~g}$ " $>0,0212 \mathrm{~g} \mathrm{BaSO}_{4}, 0,002902 \mathrm{~S}$.

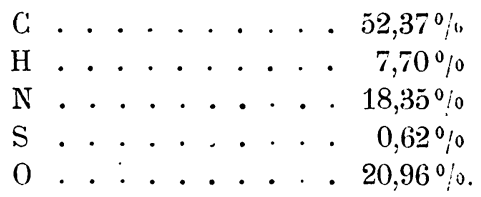

Wenn man die Werthe dieser Analyse mit denen von A. Kossel ${ }^{1}$ ) und Lilienfeld ${ }^{1}$ ) angegebenen mittleren Werthen vergleicht, so findet man eine nahe Uebereinstimmung.

Das von mir erhaltene Histon.
C. . . $52,37 \%$
H. . . $7,70 \%$ $52,31 \%$ $52,34 \%$
N . . . $18,35 \%$
$7,20 \%$
$18,46 \%$
S. . $0,62 \%$
$0,50 \%$
$7,31 \%$

Histon v. A. Kossel.

Histon v. Lilienfeld.

Der von mir aus der Thymusdrüse neu dargestellte Körper, den ich als "Parahiston» bezeichnen will, unterscheidet sich, wie man aus den gegebenen Werthen der Analyse ersieht, deutlich von Histon, hauptsächlich durch seinen grösseren Gehalt an Schwefel.

\begin{tabular}{rr} 
Parahiston (im Mittel) & \multicolumn{1}{c}{ Histon } \\
C $. . .51,84 \%$ & $52,37 \%$ \\
H $. . . .7,93 \%$ & $7,70 \%$ \\
N $. . .17,84 \%$ & $18,35 \%$ \\
S $. . . .1,99 \%$ & $0,62 \%$ \\
$O \quad . . .20,46 \%$ & $20,96 \%$
\end{tabular}

Ferner unterscheidet sich das «Parahiston» vollständig durch die Reactionen einerseits vom Histon, anderseits vom Protamin.

Ivar Bang ${ }^{2}$ ) weist auf die Fähigkeit des Histons hin sich bei Abwesenheit von Ammoniaksalzen im Ueberschuss von

1) 1. c. Heft 6).

2) Ivar Bang, Studien über Histon (Diese Zeitschrift. Bd. XXVII, 
Ammoniak zu lösen. Die von A. Kossel ${ }^{1}$ ) angegebene Fällung durch Ammoniak tritt nach Bang nur bei Anwesenheit von Ammoniaksalzen ein.

Diese Reaction und die anderen von Ivar Bang ${ }^{2}$ ) angeführten Reactionen, wie Coaguliren beim Erhitzen und Fällen mit Salpetersäure, zeigt das von mir erhaltene "Parahiston" nicht. Es löst sich in Ammoniak sowohl bei Abwesenheit als auch Anwesenheit von Ammoniaksalzen. -

Fassen wir die Resultate obiger Untersuchung zusammen, so sehen wir, dass sich auf die beschriebene Weise aus der Thymusdrüse bei Behandlung mit Schwefelsäure zwei basische eiweissartige Körper ausziehen lassen, welche durch Ammoniak getrennt werden konnten. Der eine Körper ist im Ueberschuss von Ammoniak und Wasser unlöslich und stellt seinen Eigenschaften nach Histon dar, der andere, das "Parahiston", ist in Ammoniak und Wasser leicht löslich und unterscheidet sich in seiner Zusammensetzung und seinen Reactionen von Histon. Es ist sehr leicht möglich, dass das durch Fällung mit Alkohol und Aether erhaltene wasserlösliche Histon, welches durch Zersetzung von Nucleohiston erhalten wurde, das "Parahiston" als Verunreinigung enthalten hat. Bei der Reinigung dieses "Parahistons» scheidet sich noch ein dritter Körper ab, welcher in Alkohol sehr schwer löslich ist. Diesen Körper näheren Untersuchungen zu unterziehen, ist mir wegen der geringen Menge, in der ich ihn erhielt, nicht gelungen. Ich will hier nur bemerken, dass er die Fähigkeit besitzt, Eiweiss (Witte'sches Pepton) zu fällen.

Zum Schluss sei es mir gestattet, Herrn Professor A. Kossel meinen aufrichtigsten Dank auszusprechen für seine freundlichen Rathschläge und für die Aufmerksarnkeit, mit der er meine Arbeit verfolgte.

Marburg den 21. Juli 1899.
1) A. Kossel (l. c.)
2) I. Bang (1. c.) 